\title{
Novel designs for minimizing diffraction effects of large segmented mirror telescopes
}

James E. Harvey, James B. Breckinridge, Ryan G Irvin, Richard N. Pfisterer

James E. Harvey, James B. Breckinridge, Ryan G Irvin, Richard N. Pfisterer, "Novel designs for minimizing diffraction effects of large segmented mirror telescopes," Proc. SPIE 10745, Current Developments in Lens Design and Optical Engineering XIX, 107450L (17 September 2018); doi: $10.1117 / 12.2326923$

Event: SPIE Optical Engineering + Applications, 2018, San Diego, California, United States 


\title{
Novel Designs for Minimizing Diffraction Effects of Large Segmented Mirror Telescopes
}

\author{
James E. Harvey ${ }^{1}$, James B. Breckinridge ${ }^{2}$, Ryan G. Irvin ${ }^{1}$ and Richard N. Pfisterer ${ }^{1}$ \\ 1. Photon Engineering, LLC \\ 310 S. Williams Blvd., Suite 222 \\ Tucson, Arizona 86711
2. Breckinridge Associates, LLC and California Institute of Technology Pasadena, CA 91125

\begin{abstract}
Diffraction effects of large segmented mirror gaps and secondary mirror support struts produce diffraction peaks or flares that are a detriment to exoplanet detection. In this paper we present a detailed diffraction analysis of innovative segmented mirror concepts utilizing curved segment gaps and secondary support struts that eliminate these diffraction spikes that can obscure the faint exoplanet image. The resulting diffraction performance will be quantitatively compared to that of both monolithic circular pupils and classical hexagonally segmented mirrors. We will utilize performance-based merit functions consisting of both radial and azimuthal profiles of the resulting telescope point spread function.
\end{abstract}

Keywords: Exoplanet exploration, Large segmented mirrors, Pinwheel pupil, Diffraction effects.

\subsection{Introduction}

Direct imaging of terrestrial exoplanets is necessary if astronomers are to obtain detailed spectra of a planet's surface and atmosphere to characterize planetary evolutionary tracks and estimate the probability that the planet is capable of supporting life. The apparent angular separation between the exoplanet and its parent star is less than one-arc second. Exoplanets are much smaller in size than the parent star and are illuminated by light from that star. The ratio of the irradiance, at the entrance pupil of the observing telescope, from the planet to that from the star is between $10^{-10}$ and $10^{-11}$, depending on the size of the planet and its separation from the star. The habitable zone for life as we know it is illustrated in Figure 1 for stars of different masses, relative to our sun. Also illustrated are the radii of various exoplanet's orbits, relative the radius of the earth's orbit.

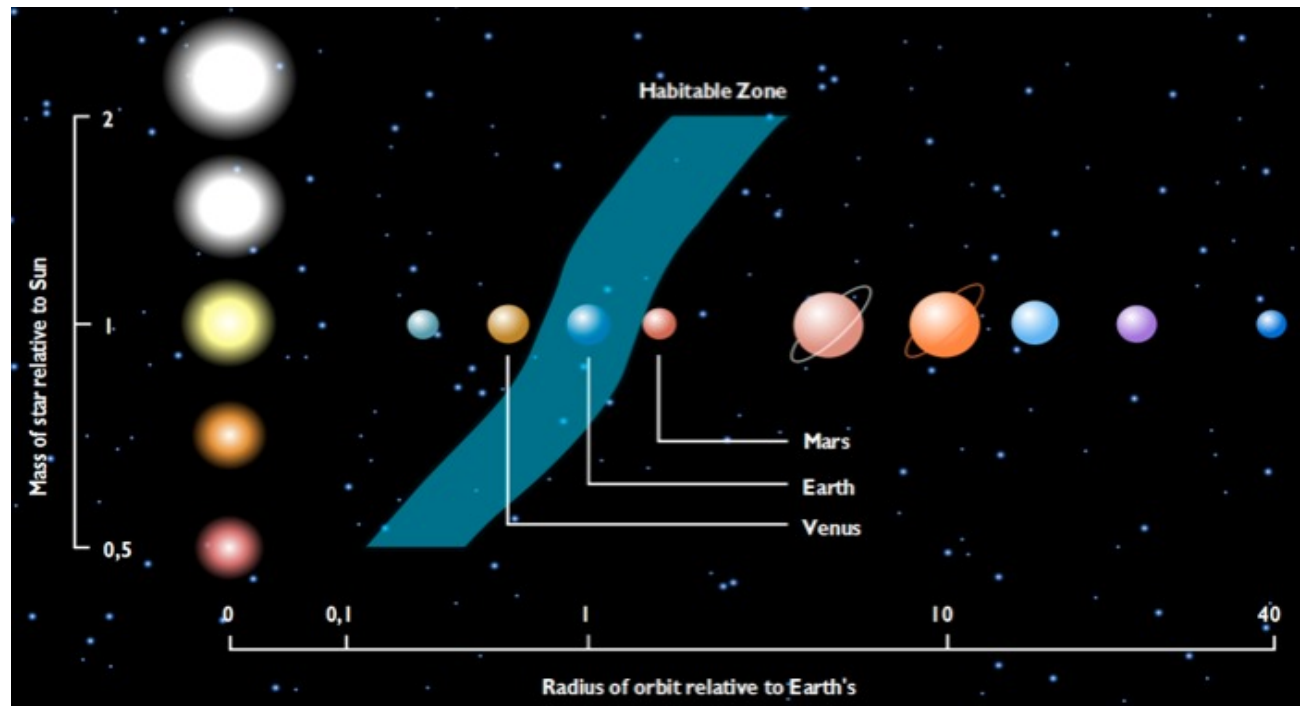

Figure 1. The habitable zone for life as we know it, displayed graphically for various sizes of stars and radii of the exoplanet's orbit.

That direct imaging and characterization of exoplanets from an Earth-orbiting space telescope is a very challenging problem is indicated in by the information provided by Table 1 and Figure $2,{ }^{1}$ which will now be discussed in turn. 
Table 1 tabulates where, with-in the telescope field-of-view (centered on a given star), an Earth-twin exoplanet might be located as a function of the distance of its host star. Column 2 shows the apparent angular separation between the host star and its terrestrial exoplanet at elongation in its orbit. Also tabulated in column 3 is the telescope aperture diameter needed to just resolve this separation. Column 4 gives the telescope aperture needed to place the exoplanet at the third diffraction ring of the host star image. In both cases the telescope apertures are calculated for 500-nm wavelength. ${ }^{1}$

Table 1. Where the Earth-twin exoplanets are, and what telescope diameter is necessary for detection.

\begin{tabular}{|c|c|c|c|}
\hline $\begin{array}{c}\text { Distance } \\
\text { Parsecs } \\
\text { PC }\end{array}$ & $\begin{array}{c}\text { Angle } \\
\text { between star } \\
\text { \& Earth twin } \\
\text { in milli-arc - } \\
\text { sec }\end{array}$ & $\begin{array}{c}\text { Aperture in } \\
\text { meters } \\
\text { Diffraction } \\
\text { limited at 500nm }\end{array}$ & $\begin{array}{c}\text { Aperture in } \\
\text { meters third } \\
\text { Airy diffraction } \\
\text { ring }\end{array}$ \\
\hline 10 & 100.0 & 1.2 & 3.7 \\
\hline 20 & 50.0 & 2.5 & 7.5 \\
\hline 30 & 33.3 & 3.7 & 11.1 \\
\hline 40 & 25.0 & 5.0 & 15.0 \\
\hline 50 & 20.0 & 6.2 & 18.6 \\
\hline 60 & 16.7 & 7.4 & 22.2 \\
\hline 70 & 14.3 & 8.7 & 26.1 \\
\hline 80 & 12.5 & 9.9 & 29.7 \\
\hline 90 & 11.1 & 11.1 & 33.3 \\
\hline 100 & 10.0 & 12.0 & 36.0 \\
\hline
\end{tabular}

Figure 2 shows a Smithsonain Astrophysics Observatory (SAO) solar system modal at a distance of 10 parsecs; i.e., 32.6 light years. It indicates that the ratio of the irradiance, at the entrance pupil of the observing telescope, from the planet to that from the star is approximately $10^{-10}$ in the visible, and $10^{-6}$ in the infrared. Visible spectra are required to provide evidence of a life-supporting environment. Exoplanets are very faint and their optical spectra are contaminated by the spectrum of the parent star. High angular resolution provided by large apertures is needed to distinguish between the spectrum of the exoplanet and its star. Thus space telescope apertures of 15 to 30 meters are needed to provide the angular resolution and the radiation-gathering power to produce a significant statistical sample of terrestrial exoplanets for analysis.

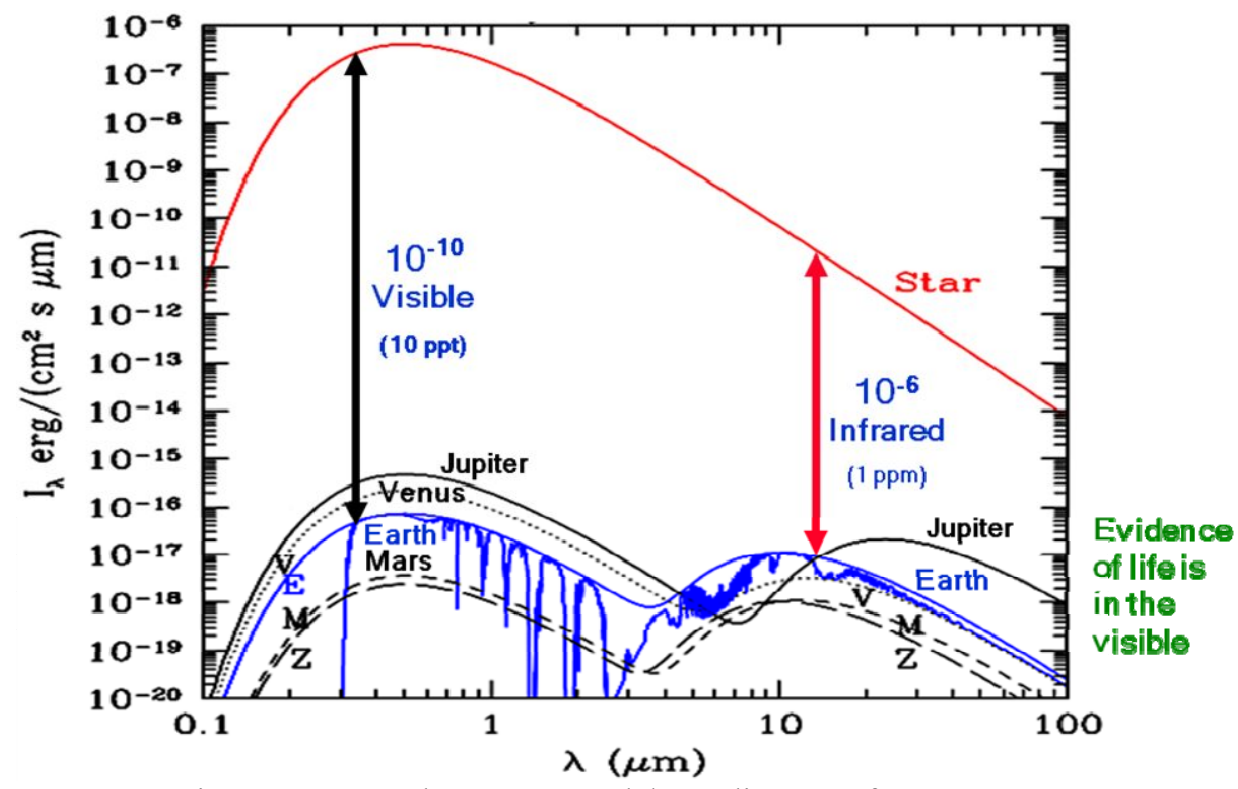

Figure 2. SAO solar system modal at a distance of 10 parsecs. 


\subsection{Diffraction Effects of Telescope Secondary Mirror Spiders and Segment Gaps}

Diffraction flares from telescope secondary mirror spiders can hide exoplanets and pollute valuable spectral data. Figure 3 shows the very prominent diffraction flares, or spikes, produced by the secondary mirror struts of the Hubble Space Telescope (HST). ${ }^{1}$

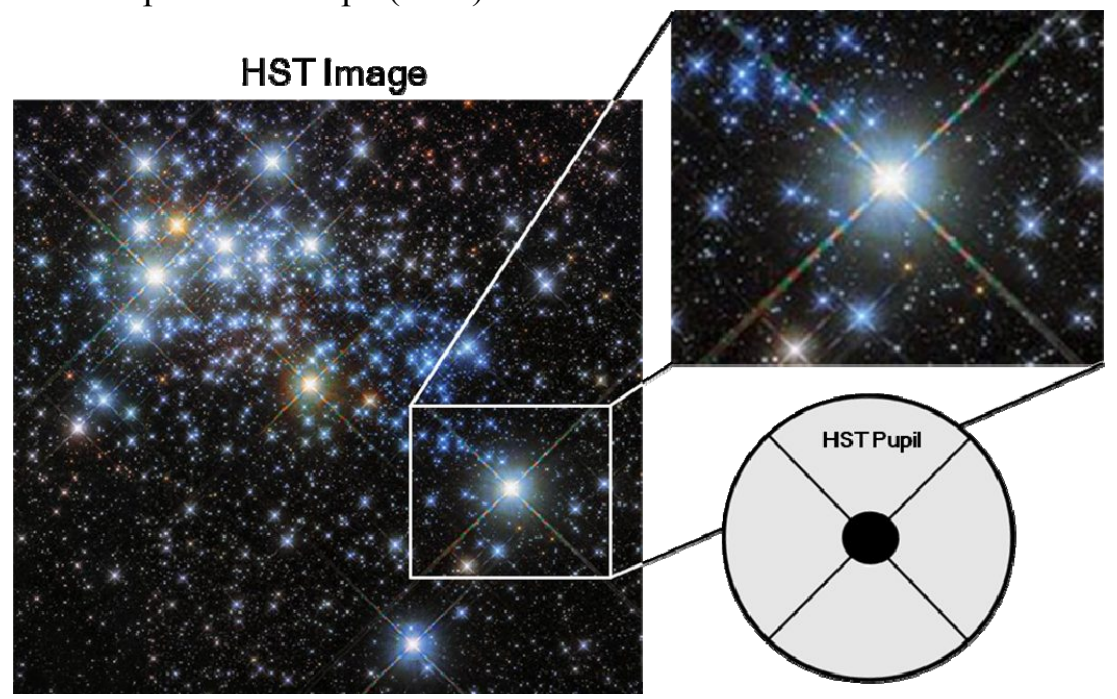

Figure 3. Illustration of diffraction flares from secondary mirror struts of the HST.

And segmented mirror gaps can produce even more diffraction flares or peaks. Figure $4 \mathrm{~b}$.) illustrates a great deal of image clutter in the form of narrow diffraction flares due to the straight secondary mirror struts and the straight sides of the triangular central obscuration of the thirty meter diameter California Extremely Large Telescope (CELT). ${ }^{2}$ One can also see the underlying discrete diffraction orders produced by the diffraction grating effects of the narrow gaps between the close-packed array of hexagonal segments making up the large primary mirror. ${ }^{2}$
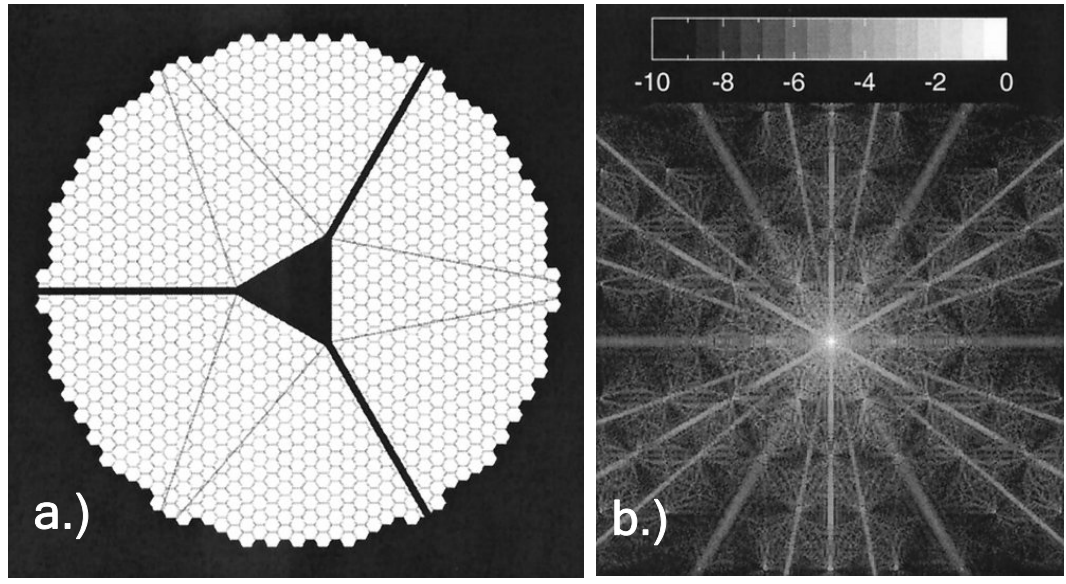

Figure 4. a.) Illustration of the proposed $30 \mathrm{~m}$ diameter pupil of the CELT, complete with segmentation geometry, central obscuration and associated secondary mirror struts; b.) Monochromatic logarithmic PSF $(\lambda=1 \mu \mathrm{m})$ for the CELT pupil. Diffraction effects of the triangular central obscuration and the secondary mirror struts are readily apparent, as is the characteristic hexagonal symmetry of the mirror perimeter and the intersegment gaps.

Yaitskova and colleagues performed an analytical study of the diffraction effects produced by extremely large close-packed hexagon segmented telescope mirrors. ${ }^{3}$ Figure 5 shows their predictions of discrete diffracted orders from the diffraction grating effects of the narrow gaps between the close-packed array of 
hexagonal segments. These diffraction peaks populate the image field of the telescope with a myriad of "fake exoplanets" from which astronomers would have to try to locate the very rare real thing.

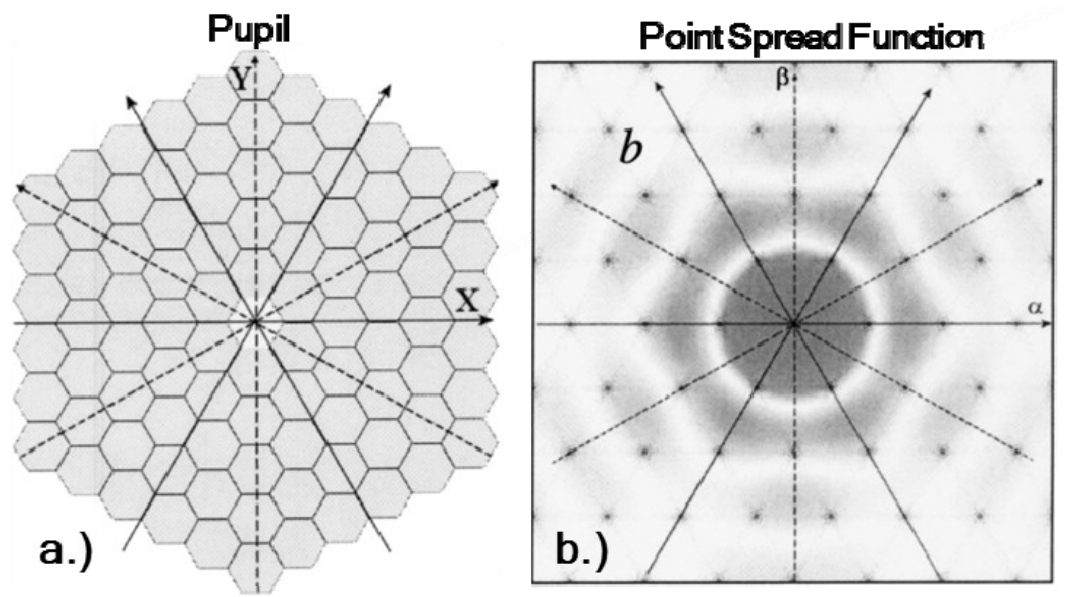

Figure 5. a.) Close-packed hexagonally segmented mirror with segmentation order $M=5$ consisting of $N=90$ segments, solid and dashed arrows illustrate the double $\pi / 3$ symmetry of the system; b.) predicted diffraction peaks that populate the image field exhibit the same $\pi / 3$ symmetry as the pupil plane.

\subsection{Historical Background of using Curved Secondary Mirror Spiders to Control Diffraction Flares}

Werenskiold (1941) was perhaps one of the first (amateur) astronomers to suggest the use of curved telescope secondary mirror spiders to improve planetary observing. ${ }^{4}$ Everhart and J. Kantorski (1959) showed experimental evidence that curved secondary mirror spiders indeed significantly suppressed the discrete diffraction flares or spikes caused by straight secondary mirror spiders. ${ }^{5}$

Richter (1984) argued that if a narrow curved obscuration in the pupil is a precise arc of a circle, then the resulting diffraction flare will be fanned out into a uniform "searchlight" or "bow tie" effect. ${ }^{6}$ Thus if the secondary mirror spiders consist of identical arcs of circles properly spaced such that their diffraction fans add up to one or more complete circles, with no gaps or overlaps, there will still be diffracted light but there will be no azimuthal variation to it. He then used Fraunhofer theory to calculate diffraction effects from curved secondary mirror spiders and used photographic diffraction patterns to compare straight and curved secondary mirror spiders.

Finally, Harvey and Ftaclas (1995) showed that diffraction effects from secondary mirror spiders can significantly degrade telescope image quality; however, the degradation varies drastically with the particular image quality criterion. ${ }^{7}$ Scalar diffraction theory and Fourier techniques were applied to parametrically model the effects of spider diffraction upon the Strehl ratio, fractional encircled energy (FEE) and the modulation transfer function (MTF). Parametric performance predictions were presented as a function of central obscuration ratio, the particular spider configuration and the width of the spiders. In particular, when the FEE is the image quality of choice, a simple empirical equation was presented and validated to be remarkably accurate for arbitrary obscuration ratio and spider configuration.

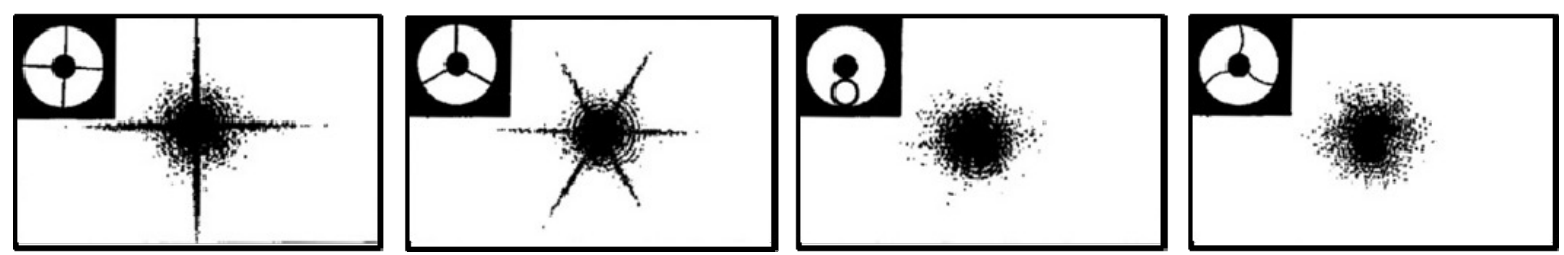

Figure 6. Diffraction effects of secondary mirror spiders upon image quality. These photographic images indicate that properly curved secondary mirror spiders can indeed effectively eliminate the narrow diffraction flares produced by straight secondary mirror spiders. 


\subsection{Lessons Learned and Insight Gained from Previous Work}

Three lessons were learned from Ref [7]. Lesson 1.): Curved secondary mirror spiders can indeed eliminate discrete diffraction flares as convincingly shown in Figure 6. Lesson 2.): Babinet's principle and Rayleigh's theorem enable parametric image degradation calculations due to diffraction effects for a variety of different image quality criteria. And Lesson 3.): The PSF from an annular aperture with opaque straight narrow secondary mirror spiders consists of an image core due to the annulus and broad diffraction flares perpendicular to the spiders. Figure 7 a.) illustrates the pupil for the case with a single narrow opaque spider, and Figure $7 \mathrm{~b}$.) shows a schematic illustration of the resulting PSF (as an image and in profile) as the sum of the two distinct parts. Since the spider has a rectangular cross-section, the resulting wide narrow diffraction flare will have the form of a sinc $c^{2}$ function as shown in Figure 7 b.).

The valuable insight comes from the realization that the composite diffraction pattern will be given by the squared modulus of the sum of the complex amplitude of Part A and Part B, and this will result in an interference pattern between the two parts where they overlap with relatively similar amplitudes.

There are thus three areas of interest in the resulting compound Fraunhofer diffraction pattern (or telescope PSF): ( $i$ ) the central portion, is dominated by the light diffracted by the annulus; ( $i i)$ the wide-angle portion, is dominated by the light diffracted by the spider, and (iii) the intermediate portion often produces an intricate interference pattern between Part A and PartB. Figure 8b.) dramatically demonstrates this intermediate region in the PSF produced by the high obscuration ratio annular aperture with four narrow opaque straight spiders illustrated in Figure 8a.).

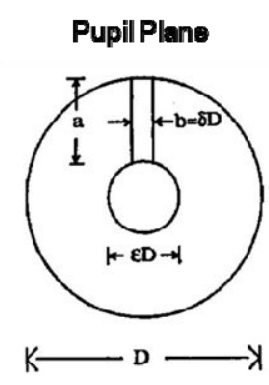

Figure 7 a.) An annular aperture with a single narrow opaque straight spider, and b.) the PSF consists of two parts, an image core and a diffraction flare perpendicular to the spider.
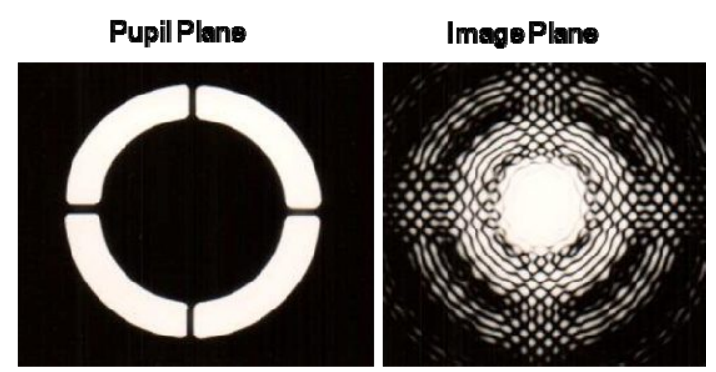

Figure 8 a.) Annular aperture with a 0.7 linear obscuration ratio and 4 spiders whose width is $3 \%$ of the aperture diameter, and b.) corresponding PSF clearly shows complex interference effects.

\subsection{Designing Secondary Mirror Spiders and Segmented Mirror Gaps to Eliminate Diffraction Flares}

Both References 6 and 7 showed that a uniform "searchlight" or "bow tie" diffraction flare subtends an angle equal to the arc of a circle subtended by the curved obstruction, in both directions! This is shown schematically in Figure 9. This behavior is intuitive if one approximates the narrow curve as a set of incremental straight segments
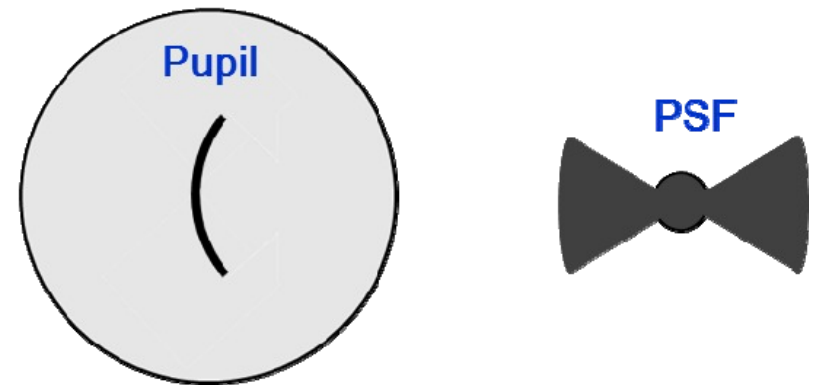

Figure 9. A narrow, curved obscuration that is an arc of a circle produces two diffracted searchlight beams emanating in opposite directions from the image core. 
We thus have two requirements for eliminating diffraction flares, or spikes, in the image plane of a telescope:

1.) We must eliminate straight lines in the pupil plane, using instead arcs of circles.

2.) And those arcs of circles, when added together in the pupil (by pure translation) must precisely constitute an exact semi-circle, or integer multiple thereof.

If these two conditions are satisfied, light diffracted from the properly curved secondary mirror spiders should be uniformly fanned out over a full $360^{\circ}$ with no overlap and no gaps. Thus there should be no variations in the azimuthal profile of the telescope PSF. This is a very desirable situation for exoplanet detection!

We will apply the above two conditions to an annular mirror with three straight secondary mirror spiders as shown in Figure 10(a). Color-coding the three struts, and indicating a $60^{\circ}$ angle centered on the left side of the horizontal diameter of the annular mirror results in Figure 10(b). Translating a line (no rotation) the length of the vertical red strut until it exactly subtends the $60^{\circ}$ angle and constructing the $60^{\circ}$ arc of a circle which has this line as its chord results in Figure 10(c). This is a $60^{\circ}$ arc of a circle which has a radius of $(1-\varepsilon) \mathrm{D} / 2$, where $\mathrm{D}$ is the diameter of the annular mirror and $\varepsilon$ is the linear obscuration ratio. Place two more $60^{\circ}$ arcs on that circle at $120^{\circ}$ intervals as shown in Figure 10(d). Now translate (again no rotation) the red arc to the previous position of the red strut as indicated by the red arrows in Figure 10(e). Likewise translate the blue arc to the previous position of the blue strut as indicated by the blue arrows in Figure 10(f). Finally translate the green arc to the previous position of the green strut as indicated by the green arrows in Figure $10(\mathrm{~g})$. The resulting three curved secondary mirror spiders shown in Figure 7(h) strictly satisfies the above conditions.

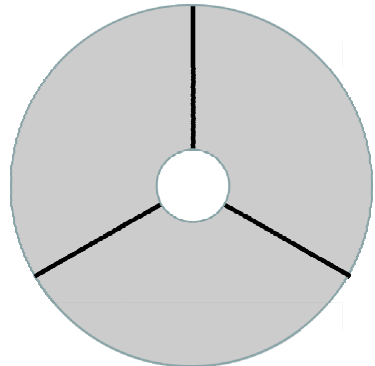

(a)

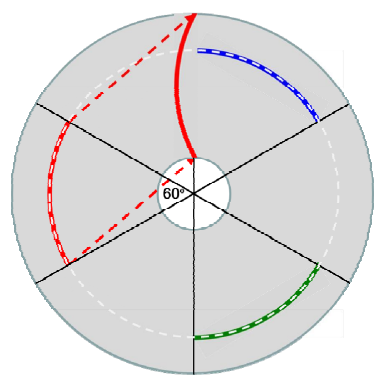

(e)

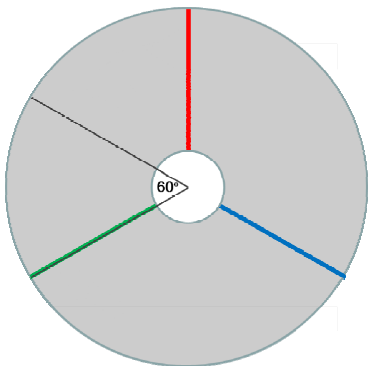

(b)

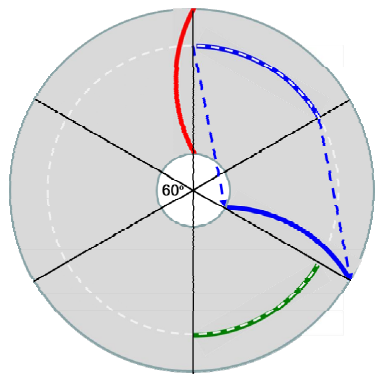

$(f)$

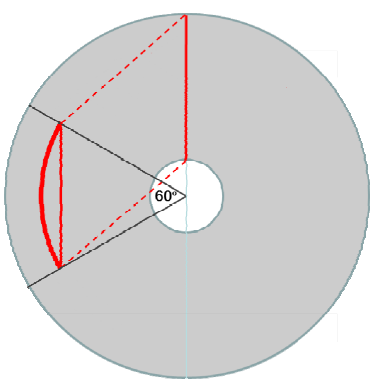

(c)

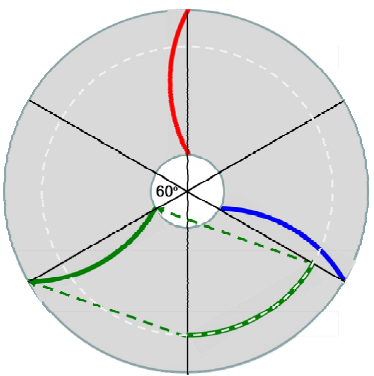

$(\mathrm{g})$

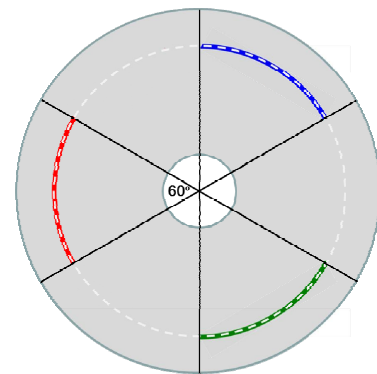

(d)

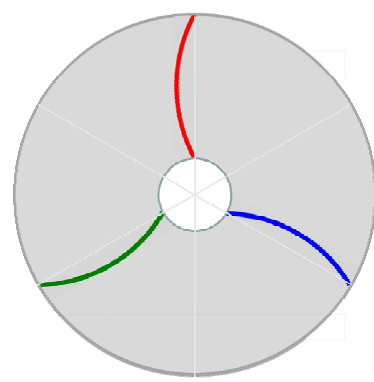

(h)

Figure 10. Illustration of one procedure to convert three straight secondary mirror spiders to three curved spiders satisfying the two requirements listed above.

Detailed diffraction analysis of these curved secondary mirror spiders showing the extent to which the undesirable diffraction flares caused by straight spiders are suppressed will be presented in the next section.

Using the "Keystone" and "Piewedge" segmented mirror configurations for large space-based telescopes, ${ }^{8}$ we will now illustrate "Pinwheel Pupil" concepts for increasingly large segmented mirror telescope diameters for which both the secondary mirror spiders and all of the segmented mirror gaps will consists of arcs of circles which satisfy the two conditions expressed at the top of this page. 
Figure 11 illustrates a ten-meter diameter 3 Tier Piewedge 12 annular segmented mirror concept with a linear obscuration ratio of $\varepsilon=0.25$. The primary mirror consists of 36 total segments (12 in each tier). The three tiers are separated by two circular gaps at radii equal to $r_{1}=2.5 \mathrm{~m}$ and $r_{2}=3.75 \mathrm{~m}$. There are twelve radial gaps through all three tiers $\left(30^{\circ}\right.$ arc of a circle). Six of these radial gaps are obscured by secondary mirror struts that cast a shadow over the gaps that are also $30^{\circ}$ arcs of a circle. The segment gaps will be approximately $20 \mathrm{~mm}$ wide, and the secondary mirror struts will be somewhat wider to provide the necessary mechanical support.

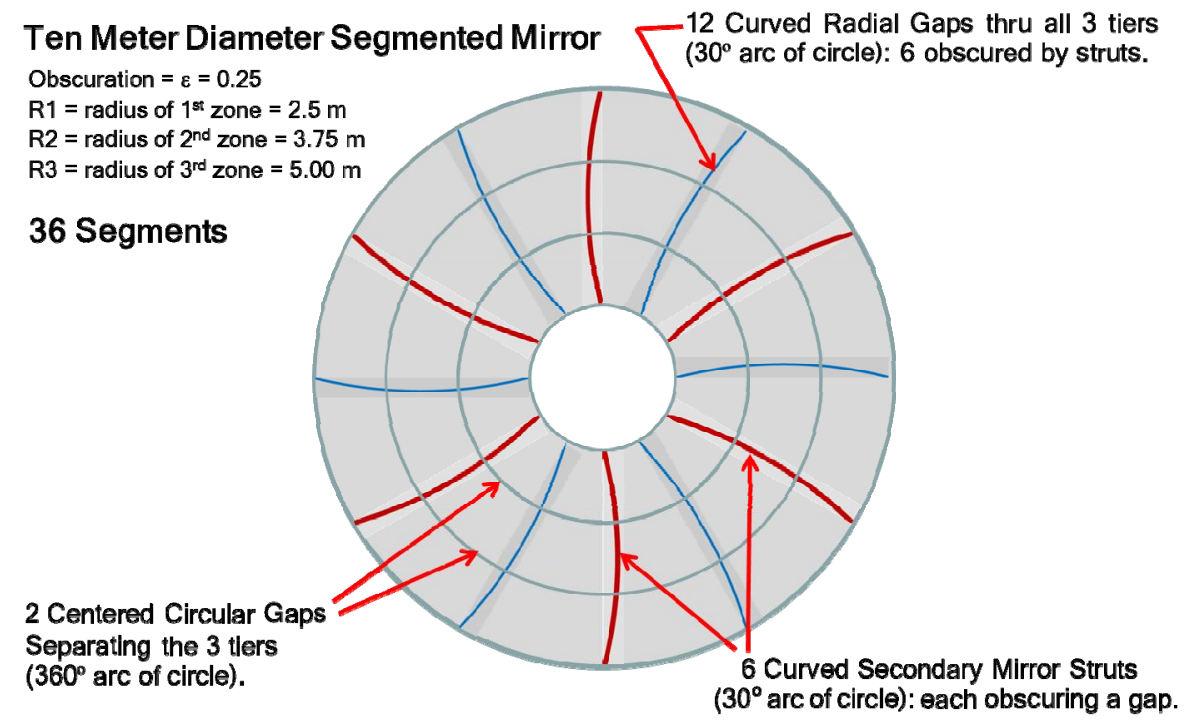

Figure 11. Illustration of the "Pinwheel Pupil" concept for a ten-meter diameter segmented primary mirror for a space-based telescope for exoplanet exploration. The curved segmented mirror gaps and secondary mirror struts are intended to suppress undesirable diffraction effects.

The 3 Tier Keystone 24 segmented mirror configuration for a 20 meter diameter annular segmented mirror concept with all segment gaps and secondary mirror spiders made up of arcs of circles satisfying the two conditions at the top of page 4 is shown in Figure 12.

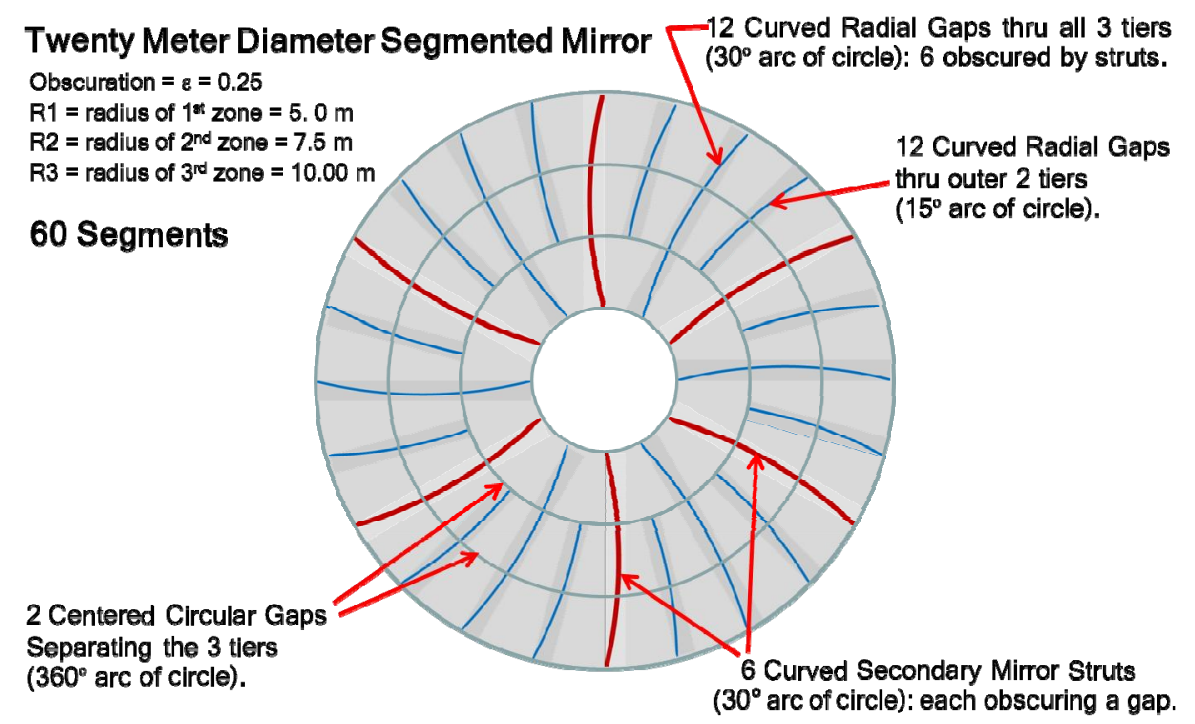

Figure 12. Illustration of the "Pinwheel Pupil" concept for a twenty-meter diameter segmented primary mirror for a space-based telescope for exoplanet exploration. The curved segmented mirror gaps and secondary mirror struts are intended to suppress undesirable diffraction effects. 


\subsection{Diffraction Analysis of Curved Secondary Mirror Spiders and Segmented Mirror Gaps}

In this section we will apply modern computation tools ${ }^{9}$ to perform detailed parametric numerical diffraction calculations to provide insight and demonstrate the extent to which discrete diffraction flares from straight secondary mirror spiders and segmented mirror gaps can be minimized or eliminated. As an ideal reference point, Figure 13 illustrates an unobscured circular pupil function, the corresponding PSF (log irradiance, $10^{-6}$ floor), and the radial and azimuthal irradiance profiles of that PSF. Much of this Section was previously discussed in Ref [10].

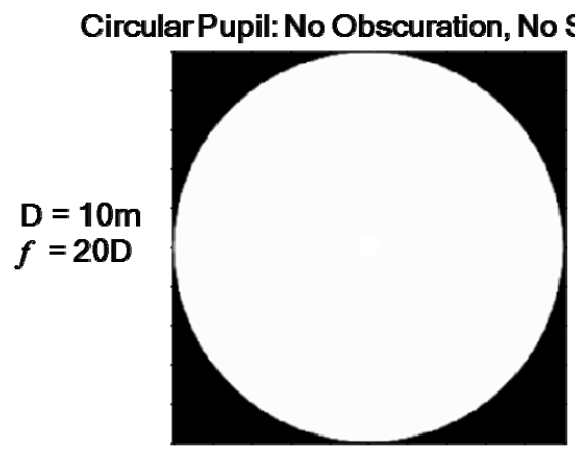

Color-coded PSF calculated by FRED.
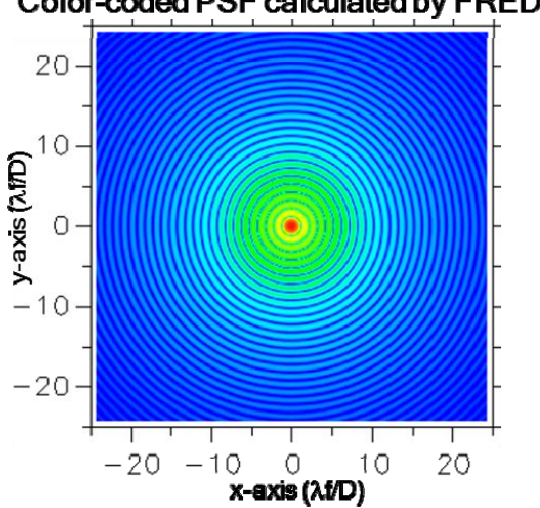
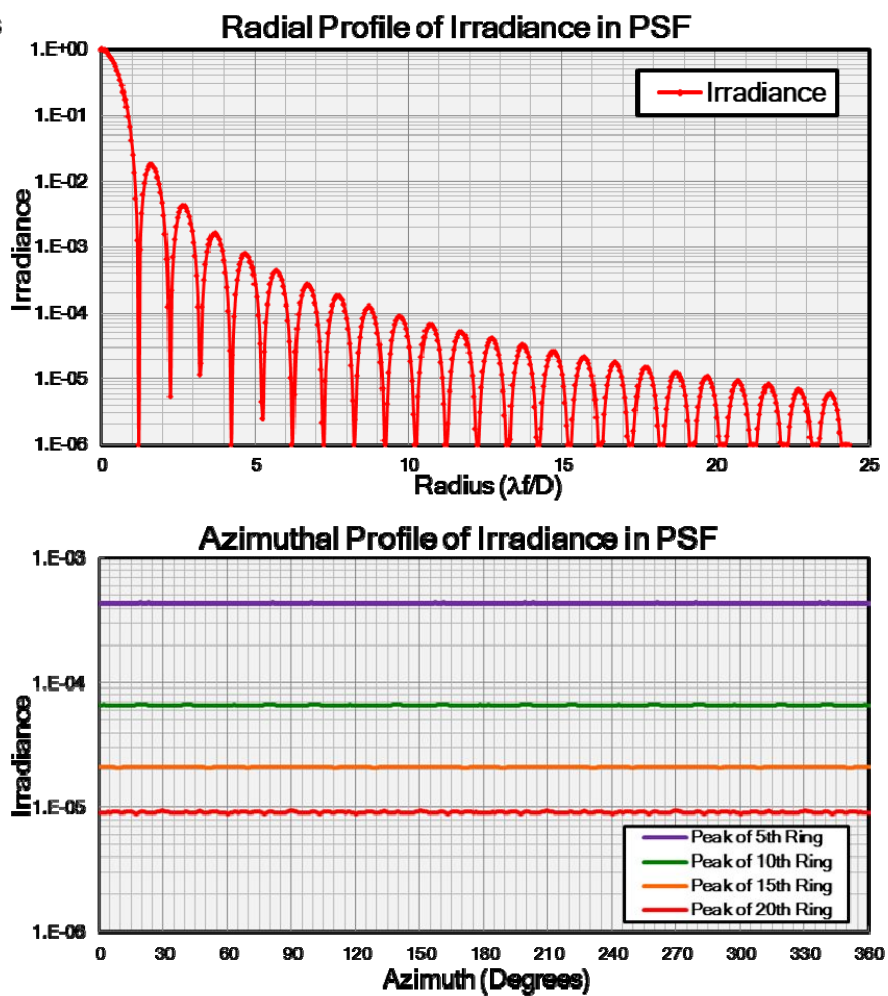

Figure 13. The clear circular pupil function, corresponding rotationally symmetric PSF, and both the radial and azimuthal irradiance profiles of the PSF are illustrated for a telescope with an unobstructed circular aperture.

Note that this ideal PSF consists of a central image core surrounded by the familiar concentric diffraction rings characteristic of a circular clear aperture. The peaks of the diffraction rings are monotonically decreasing and their spacing also slowly decreases monotonically, according to the well-known Airy pattern. ${ }^{11}$ The azimuthal irradiance profiles are indicated at the peak of the $5^{\text {th }}$ diffraction ring, as well as the $10^{\text {th }}, 15^{\text {th }}$ and $20^{\text {th }}$ ring, thus spanning the habitable zone for exoplanets. There is only a slight (perhaps numerical) irregularity on these azimuthal profiles.

Figure 14 illustrates the pupil function, PSF, and the radial and azimuthal irradiance profiles of the PSF for an annular pupil with a linear obscuration ratio of $\varepsilon=0.2$. Note that the central image core is still surrounded by the concentric diffraction rings; however, the peaks in the radial profile are no longer monotonically decreasing due the central obscuration. The azimuthal profiles are still nice and uniform.

Figure 15 illustrates the pupil function, PSF, and the radial and azimuthal irradiance profiles of the PSF produced by an annular pupil with a linear obscuration ratio of $\varepsilon=0.2$ and three straight secondary mirror spiders whose width is equal to $1 \%$ of the primary mirror diameter. Note that there are six discrete diffraction flares as expected; however, as these narrow diffraction flares cross the concentric diffraction rings from the annulus very pronounced interference effects occur which are dramatically exhibited by the azimuthal profiles. 


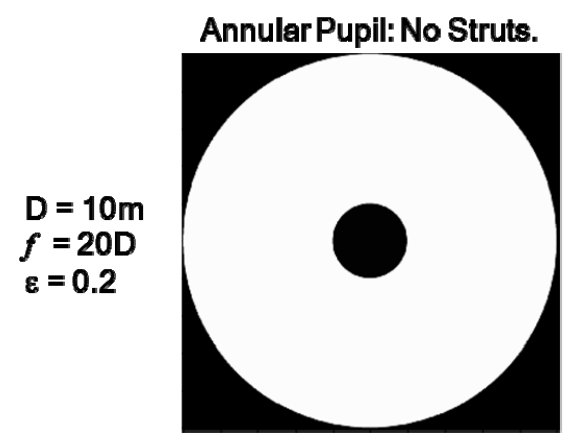

Color-coded PSF calculated by FRED.

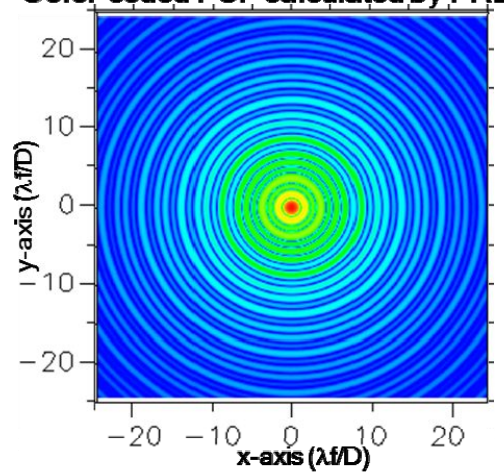

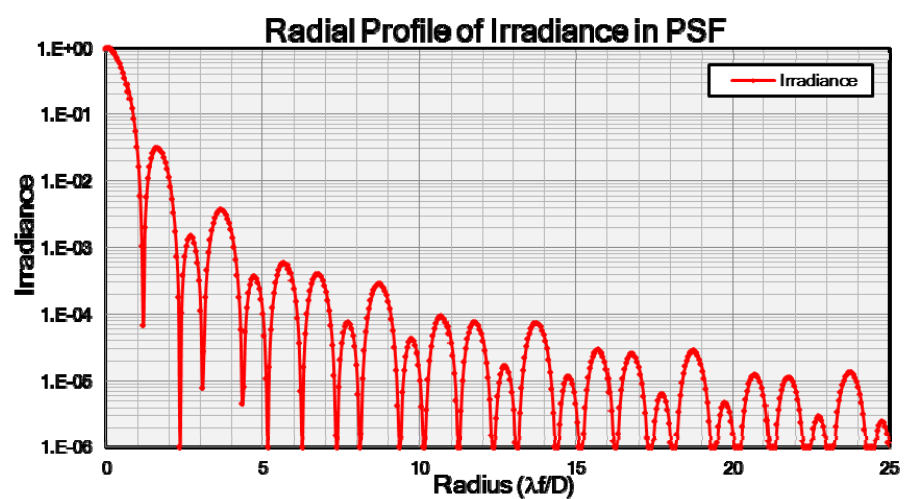

Azimuthal Profile of Irradiance in PSF

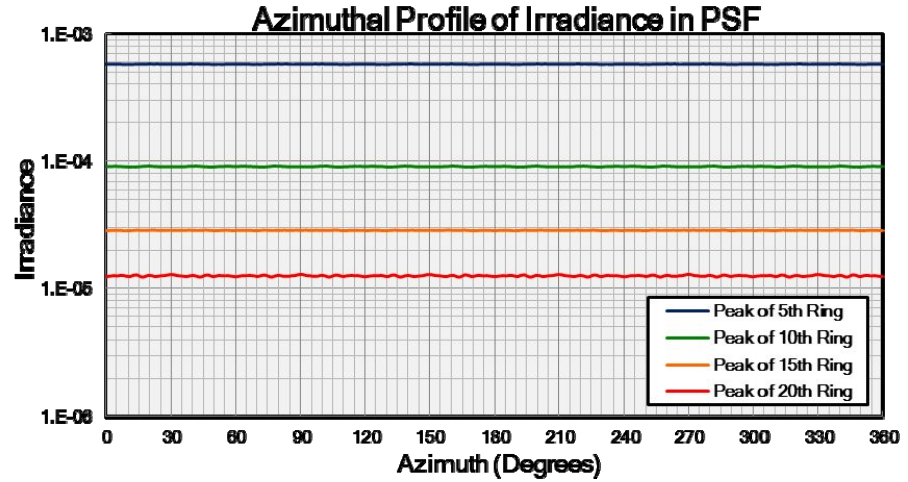

Figure 14. The annular pupil function, corresponding rotationally symmetric PSF, and both the radial and azimuthal irradiance profiles of the PSF are illustrated for a telescope with an annular aperture.

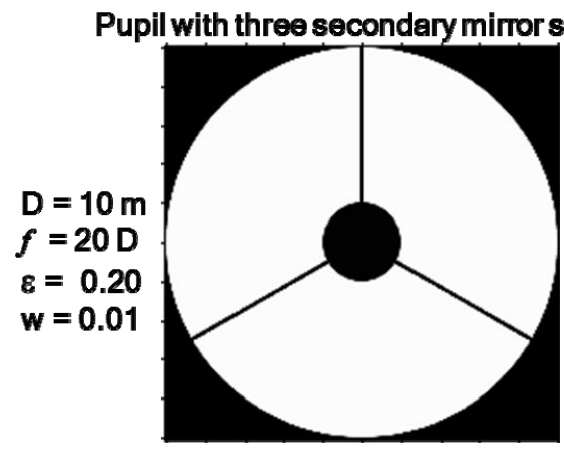

Color-coded PSF calculated by FRED.

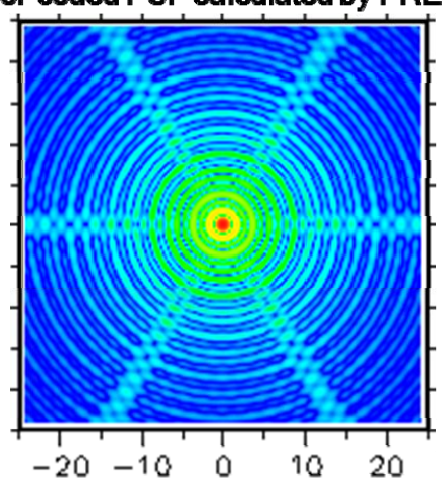

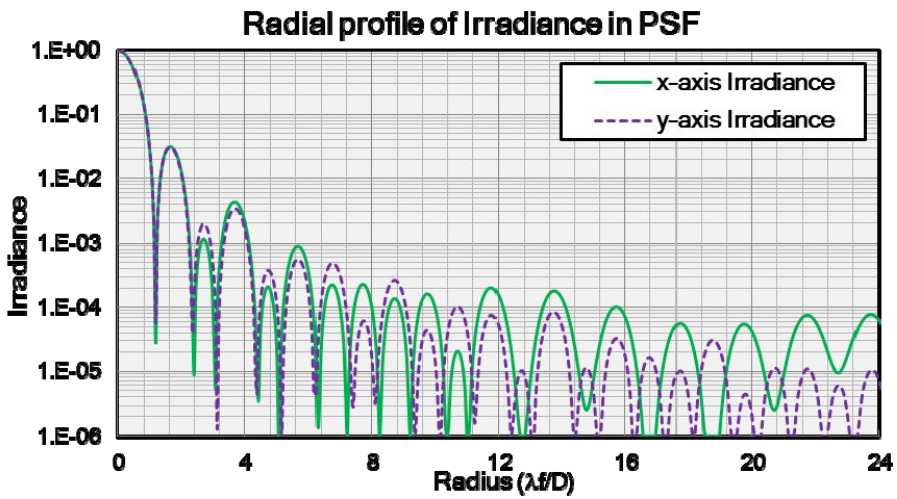

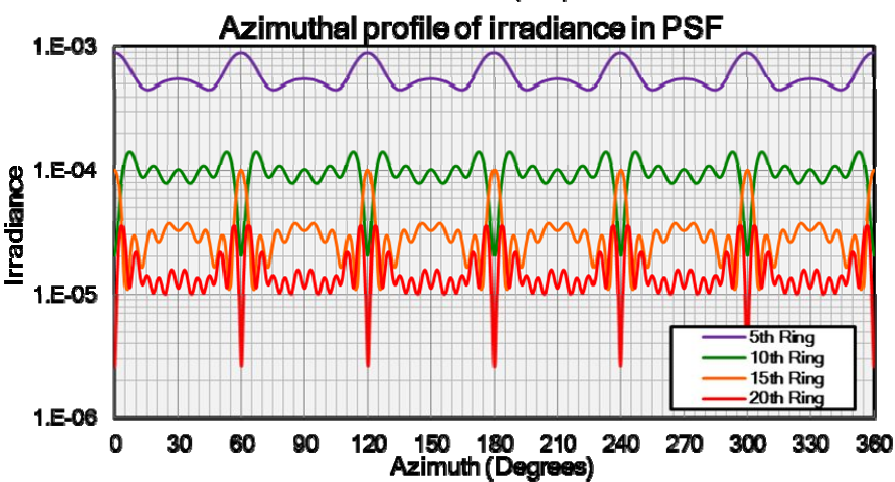

Figure 15. The pupil function, corresponding PSF, and both the radial and azimuthal irradiance profiles of the PSF are illustrated for a telescope with an annular aperture with three straight secondary mirror struts. 
In order to gain insight into the diffraction effects of straight secondary mirror spiders, Figure 16 illustrates the pupil function for an annular aperture $(\varepsilon=0.16)$ and three straight secondary mirror spiders whose width relative to the aperture diameter becomes smaller and smaller. Simulated diffraction PSFs are shown for each case out to approximately 50 diffraction rings (middle row) and out to approximately 12 diffraction rings (bottom row).

The amount of radiant power diffracted out of the image core is proportional to the area of the secondary mirror spiders, and since the struts have a rectangular cross-section, the individual diffraction flares are sinc ${ }^{2}$ functions. ${ }^{7}$ As the width of the spider is reduced there is less and less radiant power in the flares and it is being diffracted out into larger and larger angles. This behavior is plainly visible in the middle row of simulated PSFs. In the bottom row, left column, we see that the image core (diffraction pattern of the annulus) dominates the diffraction flares from the spiders for the $1^{\text {st }}$ few rings, then the very pronounced interference effects of the diffraction flare and the image core dominates the remainder of the $1^{\text {st }} 12$ rings. As the width of the spiders decrease, these interference effects reduce until (for $\mathrm{w}=0.002 \mathrm{D}$ ) the corruption of the PSF by the diffraction flares is almost negligible.

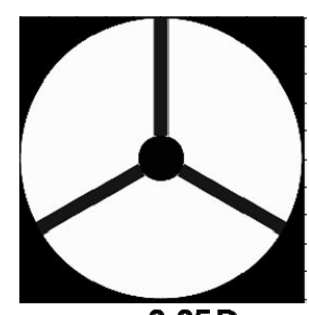

$w=0.05 D$
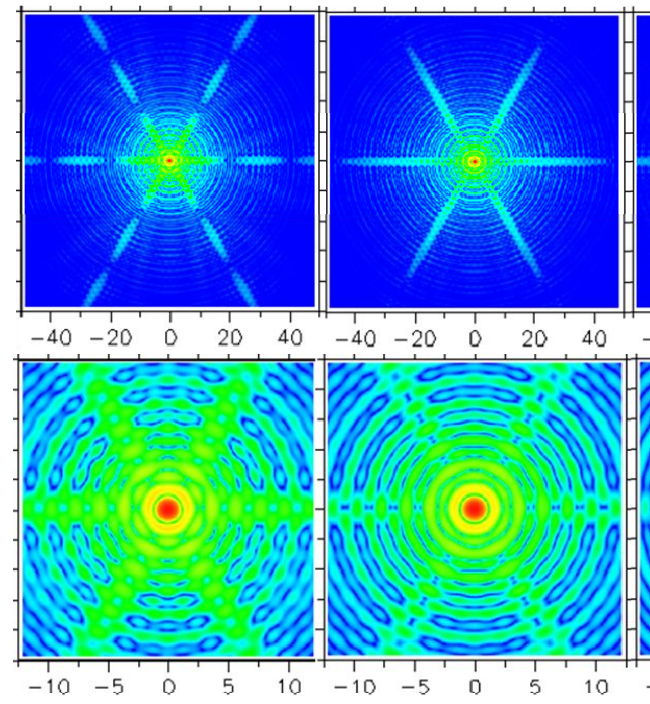

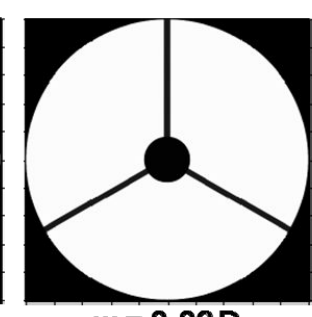

$w=0.02 D$

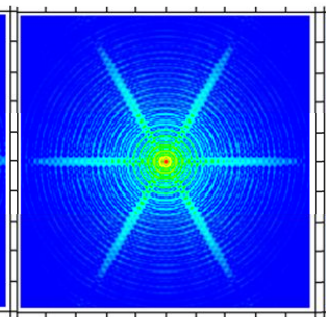

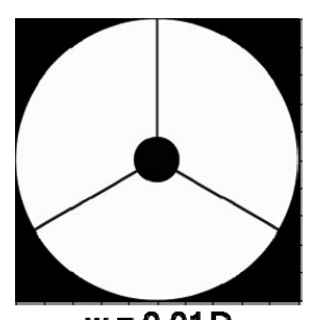

$w=0.01 \mathrm{D}$

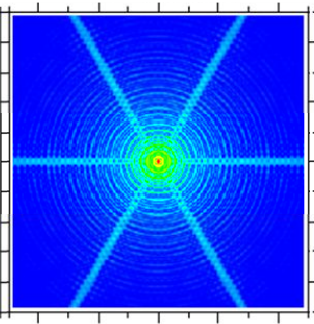

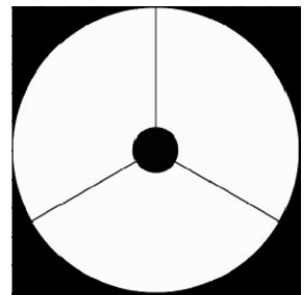

$w=0.005 D$

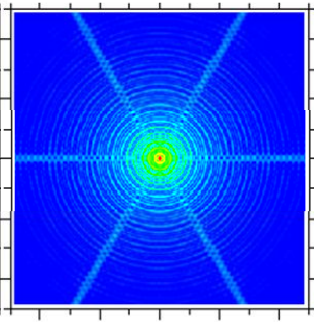

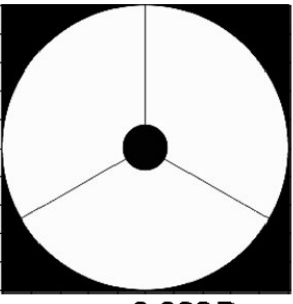

$w=0.002 D$

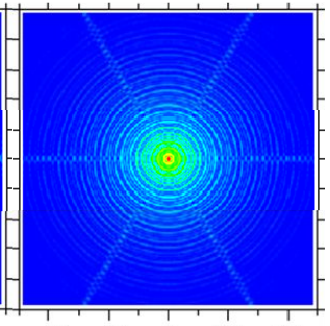

Figure 16. Simulated diffraction effects for an annular aperture $(\varepsilon=0.16)$ and three straight spiders of diminishing widths from $5 \%$ to $0.2 \%$ of the primary mirror diameter.

Figure 17 illustrates the simulated diffraction effects for an annular aperture $(\varepsilon=0.16)$ and three straight spiders $(\mathrm{w}=0.01 \mathrm{D})$ as each spider is sequentially converted to a $60^{\circ}$ arc of a circle. Simulated PSFs are again shown both out to about 50 rings and out to about 12 rings. When the vertical spider is converted to a $60^{\circ}$ arc of a circle, we see in the $2^{\text {nd }}$ PSF of the $2^{\text {nd }}$ row that the horizontal narrow diffraction flare has indeed disappeared; however, instead of a uniform "bow tie" of diffracted light we see a "bow tie" of speckles which are caused by the interference of the expected uniform "bow tie" with the concentric rings of the diffraction pattern from the annular aperture. Likewise, when the remaining two straight spiders are converted to $60^{\circ}$ arcs of a circle the corresponding discrete narrow diffraction flares disappear, but the remaining speckle pattern may be a detriment to exoplanet detection and identification. 

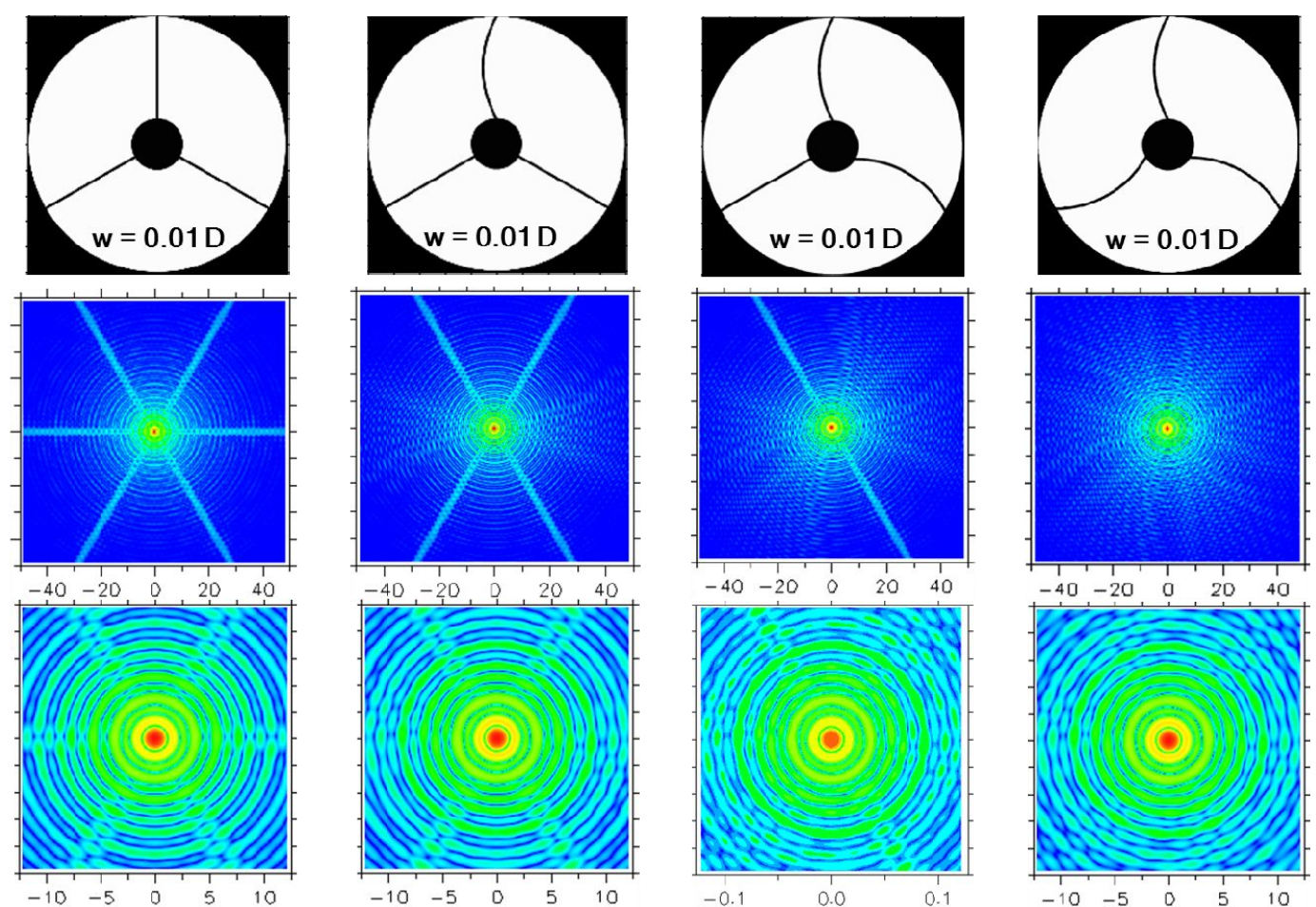

Figure 17. Simulated diffraction effects for an annular aperture $(\varepsilon=0.16)$ and three straight spiders $(\mathrm{w}=0.01 \mathrm{D})$ as each spider is sequentially converted to an arc of a circle.

However, the last column of Figure 16 indicated that when the spider width is narrowed to $\mathrm{w}=0.002 \mathrm{D}$, the corruption of the PSF due to diffraction flares were essentially negligible. And for a ten-meter diameter segmented mirror, segment gaps $20 \mathrm{~mm}$ wide are reasonable. Figures 18 to 20 thus show the image degradation (compared to an annular aperture with no spiders or gaps) due to three curved gaps whose relative width is $\mathrm{w}=0.002 \mathrm{D}$.
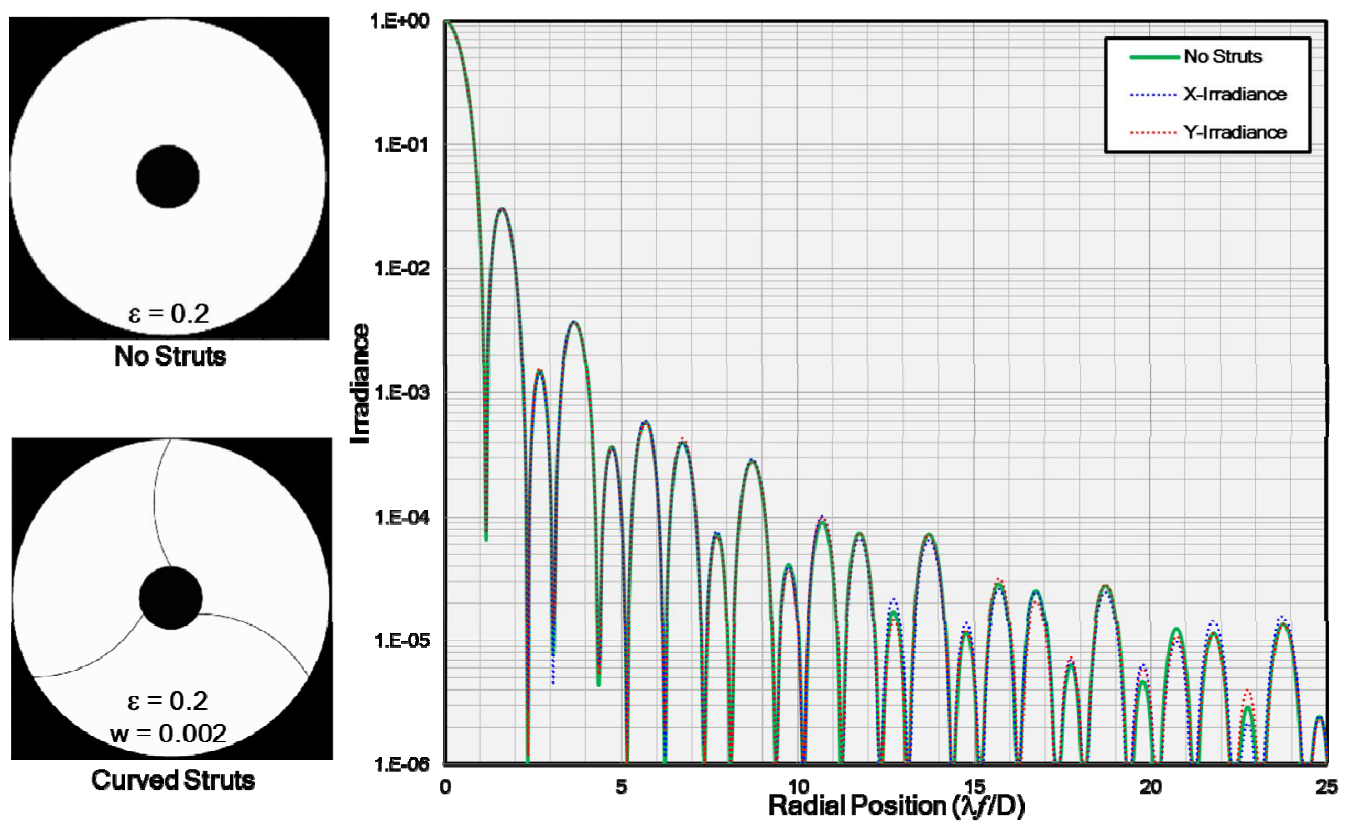

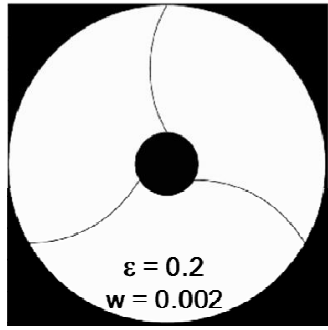

Curved Struts

Figure 18. The $\mathrm{x}$ and $\mathrm{y}$-axis radial irradiance profiles of the annular aperture with three curved spiders differ almost negligibly from each other and from that with no spiders. 

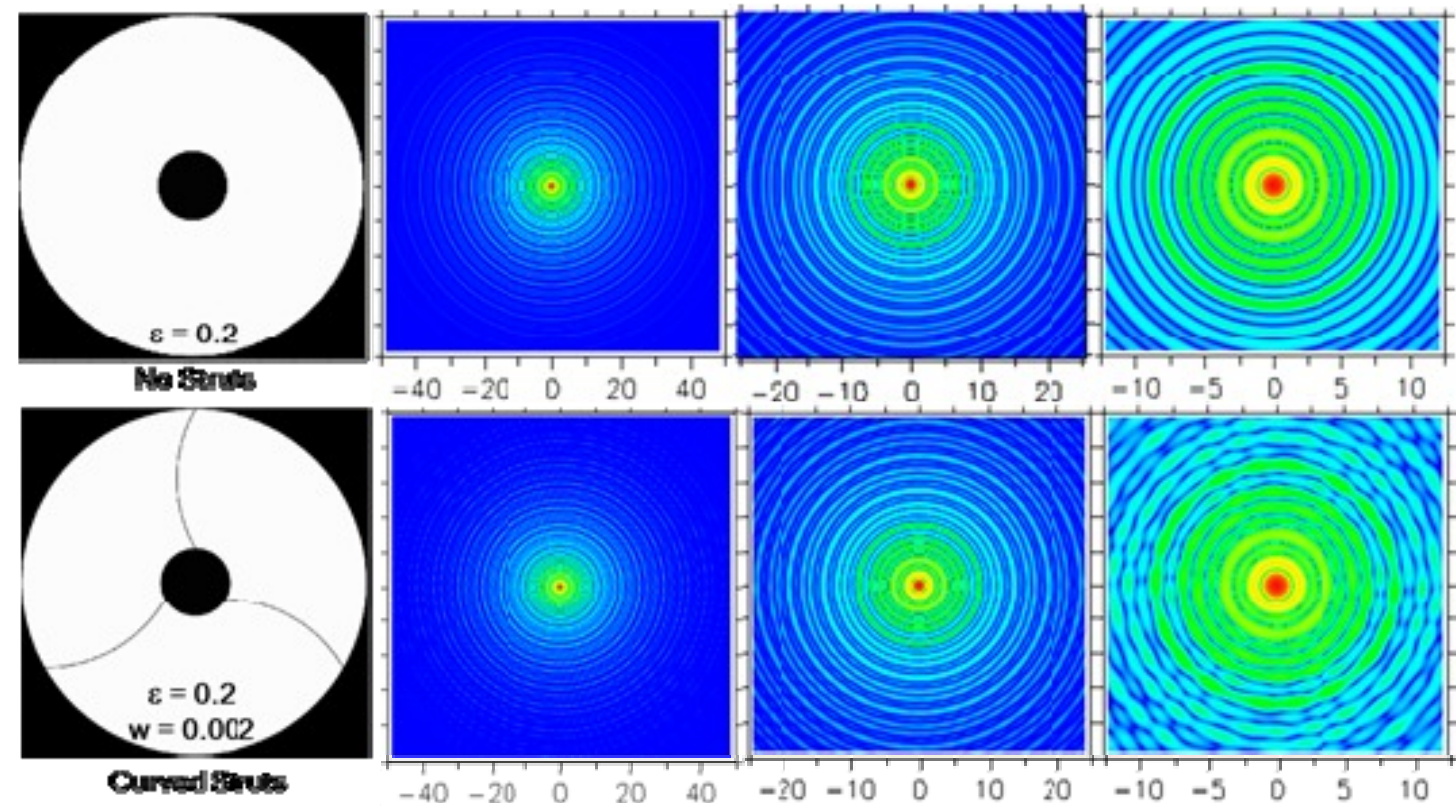

Figure 19. Simulated diffraction effects for an annular aperture $(\varepsilon=0.16)$ and three curved spiders $\left(30^{\circ}\right.$ arc of circle $)$ of relative width $\mathrm{w}=0.002 \mathrm{D}$.
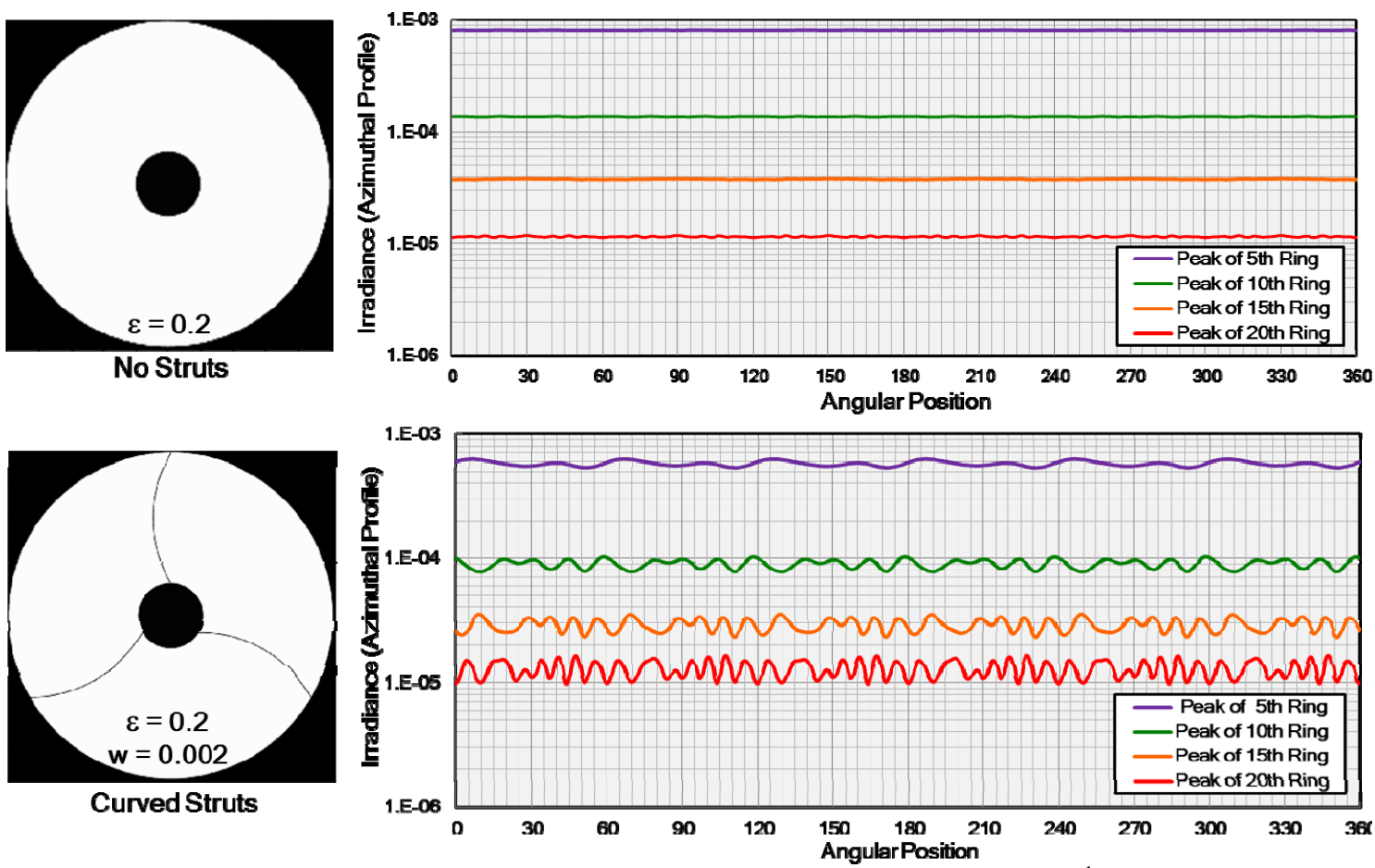

Curved Struts

AngularPosition

Figure 20. The azimuthal irradiance profiles are indicated at the peak of the $5^{\text {th }}$ diffraction ring, as well as the $10^{\text {th }}, 15^{\text {th }}$ and $20^{\text {th }}$ ring, thus spanning the habitable zone for exoplanets

The azimuthal irradiance profiles at the peak of the $5^{\text {th }}, 10^{\text {th }}, 15^{\text {th }}$ and $20^{\text {th }}$ rings (this range covers the habitable zone for most exoplanets) shown in Figure 20 for the annular aperture with three curved radial obstructions consisting of $60^{\circ}$ arcs of circles have more irregularities than we would like to see; however, it is consistent with the interference effects shown at the lower right of Figure 19 and in most of our simulations to date. 
Finally, we have modeled the diffraction effects of the 3 Tier Piewedge 12 Pinwheel Pupil concept discussed in Section 5. This is a ten meter diameter, 36 segment primary mirror with 12 curved radial gaps which are each a $30^{\circ}$ arc of a circle (thus the perceived uniform fan of diffracted light should cover the entire annulus twice over). The gap width is $\mathrm{w}=0.002 \mathrm{D}$, or $20 \mathrm{~mm}$.

The simulated PSF shown in Figure 21 is devoid of any discrete narrow diffraction flares; however, substantial interference effects are apparent. The $\mathrm{x}$ and $\mathrm{y}$-axis radial irradiance profiles are virtually identical as expected due to the bi-lateral symmetry of the pupil. The azimuthal irradiance profiles are indicated at the peak of the $5^{\text {th }}$ diffraction ring, as well as the $10^{\text {th }}, 15^{\text {th }}$ and $20^{\text {th }}$ ring, thus spanning the habitable zone for exoplanets. The azimuthal irradiance profile at the peak of the $5^{\text {th }}$ ring exhibits an almost sinusoidal variation with 12 cycles in the $360^{\circ}$. The peak-to-peak amplitude variation is about $20 \%$ of the mean value. The azimuthal irradiance profiles at the peak of the $10^{\text {th }}, 15^{\text {th }}$ and $20^{\text {th }}$ rings exhibit increasing relative peak-topeak amplitude variations and pick up additional harmonics of the 12 cycles per revolution.
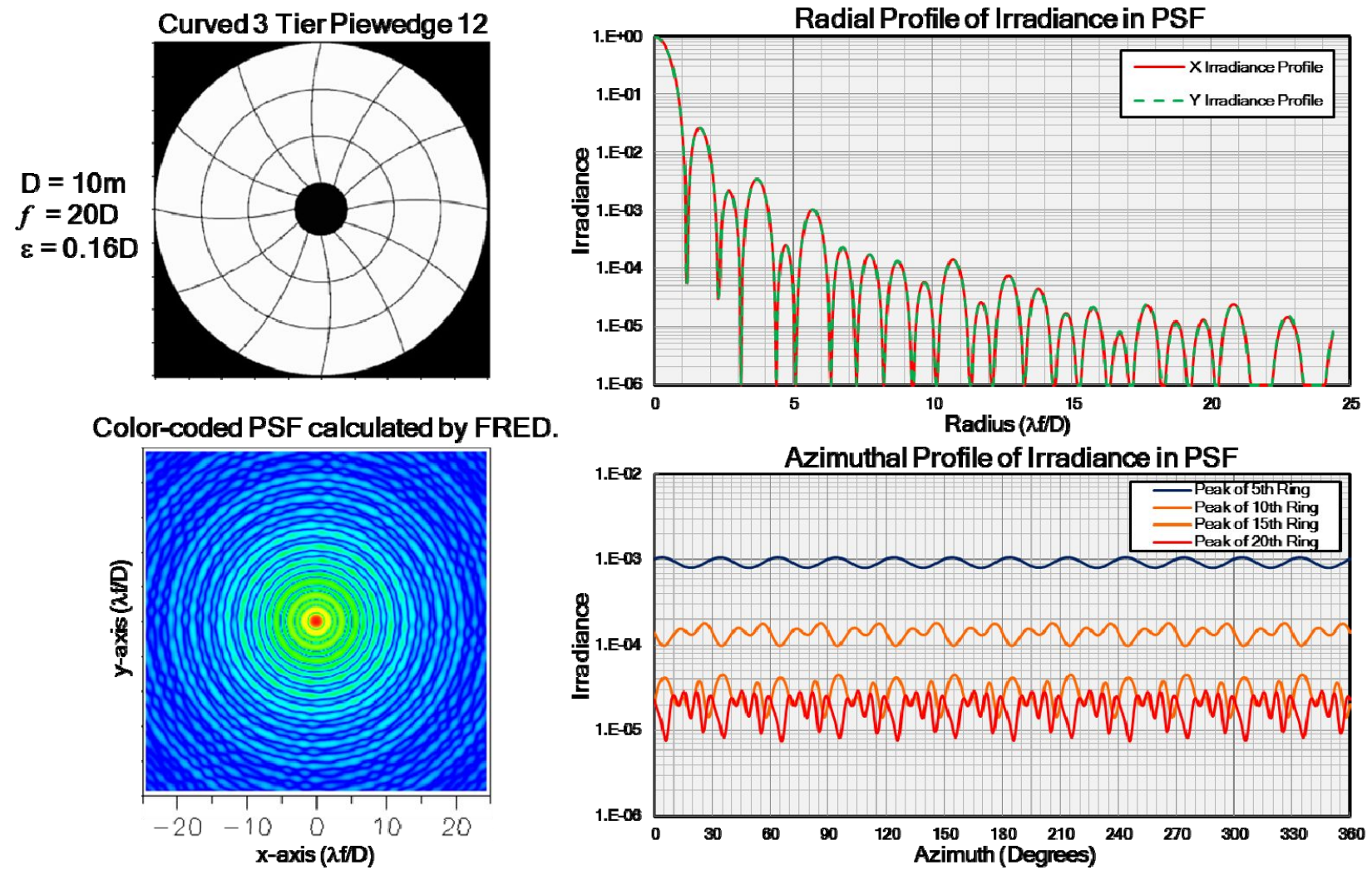

Figure 21. The 3 Tier Piewedge 12 segmented mirror configuration is illustrated with a relative gap width of $\mathrm{w}=0.002 \mathrm{D}$. The simulated PSF is devoid of any discrete narrow diffraction flares; however, substantial interference effects are apparent. The $\mathrm{x}$ and $\mathrm{y}$-axis radial irradiance profiles and several azimuthal irradiance profiles are also indicated.

\subsection{Summary and Conclusions}

We have reviewed the diffraction effects of telescope secondary mirror spiders and provided a prescription for the elimination of discrete narrow diffraction flares which calls for the elimination of straight lines in the pupil plane, using instead arcs of circles. And those arcs of circles, when added together in the pupil (by pure translation) must precisely constitute an exact semi-circle, or integer multiple thereof. We then described one way to produce the proper pupil configuration for the case of three secondary mirror spiders.

Using the "Keystone" and "Piewedge" segmented mirror configurations for large space-based telescopes, we then illustrated two "Pinwheel Pupil" concepts for increasingly large segmented mirror telescope 
diameters for which both the secondary mirror spiders and all of the segment mirror gaps consist of arcs of circles which satisfy the two conditions expressed above.

Modern computation tools were then applied to perform detailed parametric numerical diffraction calculations that provide insight and demonstrate the extent to which discrete diffraction flares from straight secondary mirror spiders and segmented mirror gaps can be minimized or eliminated.

The next step in this study might be to model the Keystone 24 pinwheel pupil for a twenty meter diameter segmented mirror telescope, and to deal with sampling and speckle (or interference) issues, perhaps by modeling polychromatic diffraction effects. Finally, an ExoPlanet coronagraph should be studied to investigate high contrast imaging issues for these pinwheel pupil designs.

\subsection{References}

[1] J B. Breckinridge and J. E. Harvey, "ExoPlanet telescope scattered light: the pinwheel-pupil solution", presented at SPIE's International Symposium on Astronomical Telescopes and Instruments, Austin, TX (June 2018). Published as Proc. SPIE 10698-61 (2018).

[2] M. Troy and G. Chanan, "Diffraction effects from giant segmented-mirror telescopes", Appl. Opt. 42 (19), 3745-3753 (July 2003).

[3] N. Yaitskova, K. Dohlen and P. Dierickx, "Analytical study of diffraction effects in extremely large segmented telescopes", JOSA A 20(8), 1563-1575 (August 2003).

[4] C. H. Werenskiold, Improved telescope spider design, J. R. Astron. Soc. Can. 35, 268-273 (1941).

[5] E. Everhart and J. Kantorski, "Diffraction effects produced by obscurations in reflecting telescopes of modest size," Astron. J. 64, 455-463 (1959).

[6] J.L. Richter, "Spider diffraction: a comparison of curved and straight legs", Appl. Opt. 23 1907-1913 (1984).

[7] J. E. Harvey and C. Ftaclas, "Diffraction Effects of Telescope Secondary Mirror Spiders upon Various Image Quality Criteria", Appl. Opt. 34, 6337-6349 (1995).

[8] S. Shaklan, "Segmented Coronagraph Design and Analysis (SCDA)", Jet Propulsion Laboratory Technical Report, (June 12, 2016).

[9] J. E. Harvey, R. G. Irvin and R. N. Pfisterer, "Modeling Physical Optics Phenomena by Complex Ray-tracing", Opt. Eng., 54(3), 035105 (2015). doi: 10.1117/1.OE.54.3.035105.

[10] J. E. Harvey, Ryan G. Irvin, Richard N, Pfisterer and James B. Breckinridge, "Diffraction Analysis of Large Segmented Mirror Concepts for ExoPlanet Exploration", presented at SPIE's International Symposium on Astronomical Telescopes and Instruments, Austin, TX (June 2018). Published as Proc. 10698-60 (2018)

[11] Eugene Hecht, Optics, 2nd Ed., Addison-Wesley Publishing Co., Reading, MA (1987). 